$\Rightarrow$ HYPERTENSION

\section{Oxidative stress and immune activation in hypertension}

Chronic vascular oxidative stress promotes immune activation and leads to vascular stiffening, ultimately resulting in renal dysfunction and hypertension, according to the findings of a recent study. "Our data suggest that common chronic conditions that promote vascular oxidation, such as diabetes, obesity, cigarette smoking and hypertension, can cause an inflammatory or autoimmune state that promotes vascular and renal disease," explains researcher David Harrison.

Stiffening of large vessels, particularly the aorta, precedes the development of hypertension and is associated with stroke, renal failure, heart failure and dementia. "Aortic stiffening commonly coexists with conditions such as ageing and diabetes via mechanisms that are poorly defined," says Harrison. "These conditions are associated with vascular oxidative stress, but exactly

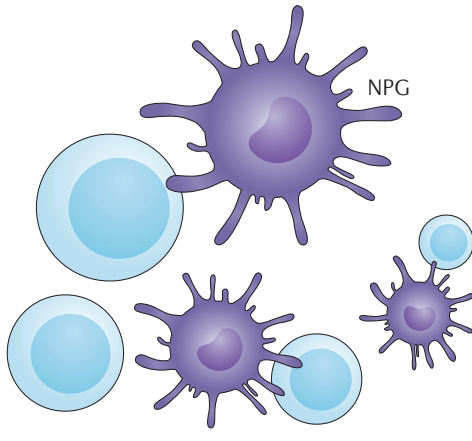

how oxidation promotes aortic stiffening and ultimately leads to hypertension is also not established."

To investigate these mechanisms, Harrison and colleagues assessed transgenic mice with chronic vascular oxidative disease; these mice developed aortic fibrosis and stiffening prior to the onset of hypertension and renal dysfunction. "We found that activated $T$ cells accumulated in the aorta of these mice at 6 months and in the kidney by 9 months," says Harrison. "The renal infiltration of $\mathrm{T}$ cells coincided with elevated blood pressure, albuminuria and a decline in the ability of the kidney to excrete a volume and sodium load."

Previous studies by Harrison and colleagues had shown that isoketals, which are formed from fatty acid oxidation, adduct to proteins in dendritic cells and act as neoantigens. Assessment of aortas from the transgenic mice revealed an accumulation of isoketals. "It was not surprising that these mice would develop isoketaladducted proteins in their vessels, but we were astonished to observe that these adducts showed up in the dendritic cells, and that they developed evidence of systemic immune activation as evidenced by increased production of cytokines such as IFN- $\gamma$ and IL-17A in T cells in the spleen," says Harrison.

Importantly, the researchers also found that treatment of transgenic mice with either the SOD mimetic, tempol, or the isoketal scavenger, 2-hydroxybenzylamine, eliminated isoketal accumulation by dendritic cells and prevented aortic fibrosis, stiffening and hypertension. Harrison believes that similar agents could prevent aortic stiffening and its untoward sequelae in humans. "We currently have support to develop new isoketal scavengers for potential use in humans," he adds.

Susan J. Allison

ORIGINAL ARTICLE Wu, J. et al. Immune activation caused by vascular oxidation promotes fibrosis and hypertension. J. Clin. Invest. http://dx.doi.org/10.1172/JCl80761 